\title{
Long-time optical transmittance measurements of silica nanofibers
}

\author{
Maha Bouhadida ${ }^{1 *}$ and Sylvie Lebrun ${ }^{1}$ \\ ${ }^{1}$ Laboratoire Charles Fabry, Institut d'Optique, CNRS, Université Paris-Saclay, 91127 Palaiseau cedex, France
}

\begin{abstract}
We perform long-time measurements of the optical silica transmittance during several months in different environments and with different nanofiber lengths. These measurements are repeatable and give guidelines to control and to improve the lifetime and the performances of the nanofiber. The dust particles on the nanofiber surface is the fundamental reason behind its degradation. Enhancing the cleanness conditions of the nanofiber environment makes its lifetime increases significantly (from some hours to some months) and enables to avoid the dramatic decrease of its transmittance even after months. The nanofiber length does not contribute to the nanofiber transmittance degradation. Stabilizing the nanofiber transmittance after its decrease is possible by putting in in a dust free box.
\end{abstract}

\section{Context and motivation}

The optical nanofiber is created by stretching a standard silica fiber until its diameter becomes typically comparable to the light wavelength. It is composed by a central part called the waist having a sub-micrometric diameter linked to the untapered end-fibers via two transition sections called tapers. The nanofiber can be easily inserted in fibered networks with very low losses which makes it significantly explored for many applications. Thanks to its small diameter, this component has a strong confinement of light inside the silica enabling the generation of nonlinear effects such as the generation of supercontinuum [1]. It has also an intense evanescent field exploited for optical sensing [2], optical traps for quantum applications [3] and the excitation of non linearities when immersed in liquids $[4,5,6]$. Despite all these attractive applications, the nanofiber performances are limited by the degradation of its transmittance during time and by its short lifetime. It has been previously demonstrated that the nanofiber transmittance degradation is mostly due to the dust particles deposited on its surface [7]. Just after pulling, the lifetime of a nanofiber placed in an environment with no particular attention to cleanliness is only some hours. In [7] the investigation of this degradation was realized using rooms with different cleanliness degrees and nanofibers with different diameters varying from $340 \mathrm{~nm}$ to $460 \mathrm{~nm}$. However, the measurements were not repeated and were performed only for one week. Besides, the possibility of stabilizing the transmittance of an already degraded nanofiber was not discussed. Only one nanofiber waist length of $1 \mathrm{~cm}$ was tested. In this work, we perform long-time measurements (over several months) of the nanofiber transmittance in environments with different levels of cleanliness. Two nanofiber waist lengths are tested $(2 \mathrm{~cm}$ and $7 \mathrm{~cm})$. We also give a simple solution to the nanofiber transmittance stabilization after its degradation. Firstly we describe the experimental setup and protocol. Then, we present the results and we discuss them giving some guidelines to preserve the nanofiber transmittance stability and to extend its lifetime.

\section{Experimental setup and protocol of measurements}

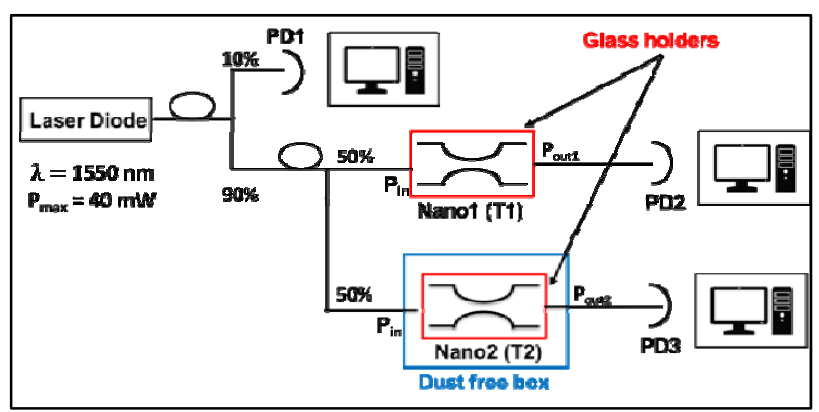

Fig. 1. Experimental setup

Fig.1 illustrates the experimental setup we build to perform the long-time measurements of nanofibers transmittance stability. This setup is totally fibered. A laser diode delivering a maximal power of $40 \mathrm{~mW}$ emits a light beam at $1550 \mathrm{~nm}$ in the $\mathrm{cw}$ regime. The beam is split by a $90 / 10$ coupler. The $10 \%$ side is used as a reference and detected by photodiode PD1. The other $90 \%$ side is split by a 50/50 coupler, delivering two beams of the same power $P_{\text {in }}$. The first beam is coupled

Corresponding author: maha.bouhadida@institutoptique.fr 
to a nanofiber (Nano1) placed in a partially protective glass holder. The second beam is coupled to a nanofiber (Nano2) placed in an identical glass holder and stored in dust free plexiglas box. Only the end-fibers connectors are out of this box. The nanofiber pulling process is described in [4]. A butane flame softens the fiber central part while two computer-controlled translation stages elongate it following the classical "pull and brush" technique. This fabrication process is performed in a class -5 clean room. The resulting nanofiber has a radius of $500 \mathrm{~nm}$. Two waist lengths are fabricated for these measurements $(2 \mathrm{~cm}$ and $7 \mathrm{~cm})$. The standard fiber is the SMF28 (ref. Corning). It is connectorized at both sides before its pulling. After fabricating the nanofiber, we partially protect it by putting it in the glass holder and transport it to the experimental setup. Our setup is built in normal lab cleanliness and humidity conditions. We check that the humidity conditions of the dust free box are the same as those of the lab. The light transmittance of the nanofiber $\mathrm{T}$ is defined as the ratio between the output transmitted power $\mathrm{P}_{\text {out }}$ and the input power $\mathrm{P}_{\text {in }}$. We denote respectively by $\mathrm{T} 1$ and $\mathrm{T} 2$ the transmittances of Nano1 and Nano2. The initial nanofibers transmittances are measured after their installation in this setup. $\mathrm{P}_{\text {out } 1}$ and $\mathrm{P}_{\text {out2 } 2}$ are detected by photodiodes PD2 and PD3. All the photodiodes acquisitions are controlled by computer. We tested two different environments of the nanofibers : the partially protective glass holder directly held in the normal lab cleanliness conditions (Environment1) and the same holder overprotected in a dust free box (Environment2). The measurements duration is about 150 days. The output power values are taken every minute, which is equivalent to 1440 samples per day. The transmittance measurements are divided in two phases : in the first phase, Nanol and Nano2 are respectively placed in Environment1 and Environment2. In the second phase, Nano1 and Nano2 are swapped. We repeat these long-time measurements several times (3 times until now) and for the two different waist lengths.

\section{Results and discussion}

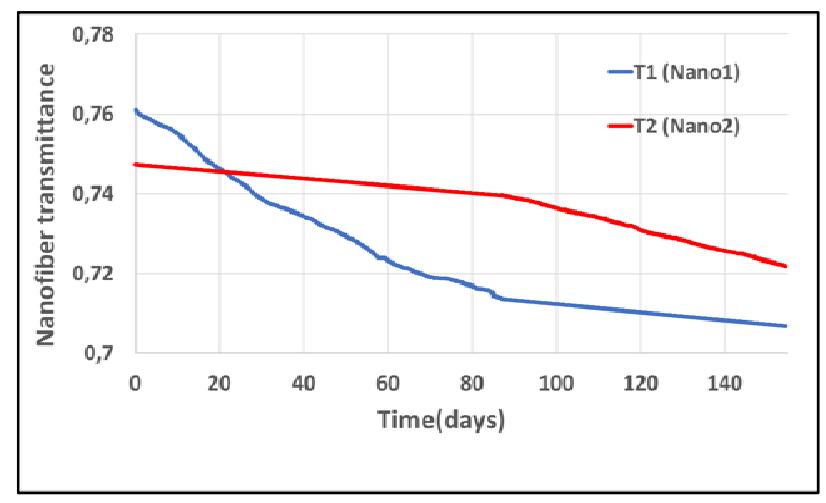

Fig. 2. Typical transmittance measurement

Fig.2 shows a typical continuous long-time transmittance measurement (about 150 days) of nanofibers having a waist length of $2 \mathrm{~cm}$. The initial transmittances are $\mathrm{T} 1=$ 0.76 and $\mathrm{T} 2=0.745$. The first phase lasted about 85 days and the second one about 65 days. The nanofibers having
$7 \mathrm{~cm}$ of length give the same trends, which proves that the waist length doesn't affect the transmittance degradation. This result is repeatable ( 3 measurements for the two tested nanofibers of different lengths). We observe that the transmittances of both Nanol and Nano2 are not dramatically degraded after 150 days as it is the case when the nanofiber is put directly in the normal lab conditions without any protection. This confirms the protection efficiency of our glass holders even if it is partial. However, the decrease of $\mathrm{T} 1$ is faster than the decrease of $\mathrm{T} 2$ in the first phase of the measurement. In environment1, T1 goes from 0.76 to $0.715\left(-5 \times 10^{-4} /\right.$ day) but $\mathrm{T} 2$ is only reduced from 0.745 to $0.74\left(-5 \times 10^{-5} /\right.$ day $)$. This slighter slope degradation illustrates the impact of the additional protection offered by the dust free box. In the second phase, we swap Nano1 and Nano2. We notice that the slope of the degradation is decreasing : $\mathrm{T} 1$ decreases from 0.715 to 0.71 in 65 days, i.e. with a slope of $-2 \times 10^{-4} /$ day (at the same time $\mathrm{T} 2$ decreases by $3 \times 10^{-4} /$ day). Then, by placing an already degraded nanofiber in a dust free box, it is possible to slow down the degradation of its transmittance. Our results also enable to predict the value of the transmittance of a nanofiber over several months, which is an important parameter for experiments.

\section{Conclusion and perspectives}

In this paper, we present long-time and repeatable measurements of nanofiber transmittances during several months, performed in different environments and with different nanofiber waist lengths. We show that the length of the nanofiber does not contribute to the transmittance degradation. By protecting the nanofiber from dust just after pulling, we are able to increase its lifetime from some hours to several months and to preserve its optical performances. Placing the nanofiber in a cleaner environment enables to slow down the degradation. These guidelines are useful to control and to enhance the lifetime and the performances of an optical nanofiber.

This work is supported by the French National Research Agency (ANR) (FUNFILM-ANR-16-CE24-0010- 03).

\section{References}

[1] M. A. Foster, A.C. Turner, M. Lipson, A.L. Gaeta, Opt. Express 16, pp. 1300-1320 (2008).

[2] L. Tong, Sensors, 18, 903 (2018).

[3] B. Gouraud, D. Maxein, A. Nicolas, O. Morin, and J. Laurat, Phys. Rev. Lett. 114, 180503(2015).

[4] L. Shan, G. Pauliat, G. Vienne, L. Tong, and S. Lebrun, Appl. Phys. Lett., 102:201110 (2013).

[5] M. Bouhadida, J. C. Beugnot, P. Delaye, K. Phan Huy and S. Lebrun, Appl. Phys. B, 125, 228 (2019).

[6] G. Fanjoux, J. Chrétien, A. Godet, K. Phan-Huy, J.-C. Beugnot, T. Sylvestre, Opt. Express, 27, 20 (2019).

[7] M. Fujiwara, K. Toubaru, S. Takeuchi, Opt. Express, 19, 9 (2011). 